\title{
Characterization of the tunicamycin gene cluster unveiling unique steps involved in its biosynthesis
}

\author{
Wenqing Chen ${ }^{1,3^{*}}$, Dongjing $\mathrm{Qu}^{1^{*}}$, Lipeng Zhai ${ }^{1}$, Meifeng Tao ${ }^{1}$, Yemin Wang ${ }^{1}$, Shuangjun Lin ${ }^{1}$, \\ Neil P. J. Price ${ }^{2 \bowtie}$, Zixin Deng ${ }^{1,3 \bowtie}$ \\ ${ }^{1}$ Laboratory of Microbial Metabolism, and School of Life Sciences \& Biotechnology, Shanghai Jiao Tong University, Shanghai \\ 200030, China \\ ${ }^{2}$ National Center for Agricultural Utilization Research (NCAUR), USDA-ARS, 1815 North University Street, Peoria, Illinois \\ 61604, USA \\ ${ }^{3}$ College of Pharmacy, Wuhan University, Wuhan 430072, China \\ $\bowtie$ Correspondence: zxdeng@sjtu.edu.cn (Z. Deng); neil.price@ars.usda.gov (N.P.J. Price) \\ Received October 18, 2010 Accepted October 26, 2010
}

\begin{abstract}
Tunicamycin, a potent reversible translocase I inhibitor, is produced by several Actinomycetes species. The tunicamycin structure is highly unusual, and contains an 11-carbon dialdose sugar and an $\alpha, \beta-1 ", 11$ '-glycosidic linkage. Here we report the identification of a gene cluster essential for tunicamycin biosynthesis by high-throughput heterologous expression (HHE) strategy combined with a bioassay. Introduction of the genes into heterologous non-producing Streptomyces hosts results in production of tunicamycin by these strains, demonstrating the role of the genes for the biosynthesis of tunicamycins. Gene disruption experiments coupled with bioinformatic analysis revealed that the tunicamycin gene cluster is minimally composed of 12 genes (tunAtunL). Amongst these is a putative radical SAM enzyme (Tun B) with a potentially unique role in biosynthetic carbon-carbon bond formation. Hence, a seven-step novel pathway is proposed for tunicamycin biosynthesis. Moreover, two gene clusters for the potential biosynthesis of tunicamycin-like antibiotics were also identified in Streptomyces clavuligerus ATCC 27064 and Actinosynnema mirums DSM 43827 . These data provide clarification of the novel mechanisms for tunicamycin biosynthesis, and for the generation of new-designer tunicamycin analogs with selective/enhanced bioactivity via combinatorial biosynthesis strategies.
\end{abstract}

KEYWORDS tunicamycin, biosynthetic gene cluster, high-throughput heterologous expression, bioassay, combinatorial biosynthesis

\section{INTRODUCTION}

Tunicamycins (Fig. 1) are a group of structurally related nucleoside antibiotics produced by several Actinomycetes species (Tsvetanova and Price, 2001; Tsvetanova et al., 2002; Price and Tsvetanova, 2007). The structure of tunicamycins consists of uracil, $\mathrm{N}$-acetylglucosamine (GlcNAc), a unique 11-carbon 2-aminodiadose sugar (tunicamine), and an $\mathrm{N}$-acyl chain with variable lengths (Tsvetanova and Price, 2001; Price and Tsvetanova, 2007). Structurally, the naturally occurring tunicamycins family antibiotics, including mycospocidins (Eckardt et al., 1975), streptovirudins (Eckardt et al., 1975; Keenan et al., 1981), MM 19290 (Kenig and Reading, 1979) and corynetoxins (Vogel et al., 1981) are all similar except the difference in the $\mathrm{N}$-acyl chain or/and replacement of 5,6-dihydrouracil for the uracil moiety (Fig. S1) (Eckardt, 1983; Price and Tsvetanova, 2007).

As a reversible, competitive translocase I inhibitor, tunicamycin targets bacterial cell wall biosynthesis by inhibition of phospho-UDP-N-acetylmuramoyl-pentapeptide translocase (MraY, Translocase I) which catalyzes an early stage of peptideglycan biosynthesis (Kimura and Bugg, 2003; Xu et al., 2004; Price and Momany, 2005; Winn et al., 2010). Unlike many other nucleoside antibiotics, tunicamycin

"These authors contributed equally to the work. 


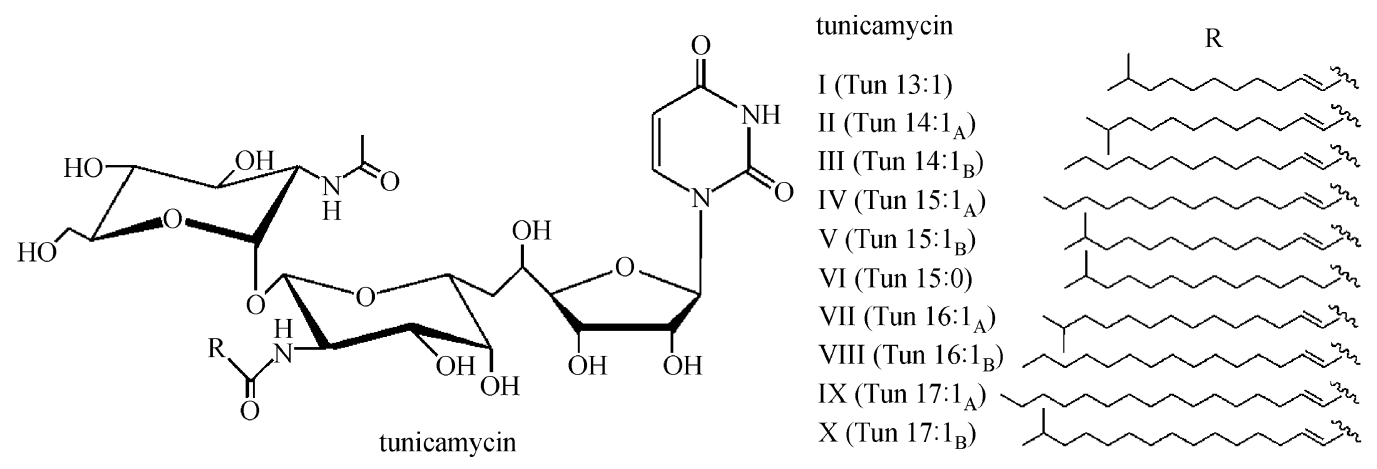

Figure 1. Chemical structures of tunicamycins. Different components are designated as tunicamycin I- $\mathrm{X}$, which correspond to components of Tun13:1-17:1 $\mathrm{B}$ as indicated in parentheses, and the latter nomination was adopted in this paper.

displayed high toxicity to mammalian, which precludes its use as antibacterial agent (Kimura and Bugg, 2003; Winn et al., 2010). This is because in eukaryotes the tunicamycins mimic the transition state analog (GlcNAc-1-P) of UDP-N-acetylglucosamine dolichol phosphate GlcNAc-1-P transferase (GPT), that catalyzes the first step in $N$-linked protein glycosylation (Winn et al., 2010). The capabilities of inhibiting the posttranslational glycosylation of proteins make tunicamycin a useful tool in the study of protein glycosylation (Winn et al., 2010). Moreover, tunicamycins have also been shown to block $N$-palmitoylation of acyl-proteins by inhibiting palmitoyltransferase (Patterson and Skene, 1994; Liang et al., 2002; Price and Tsvetanova, 2007).

Recent investigations performed with isotope feeding experiments have proposed a tentative pathway for tunicamycin biosynthesis (Tsvetanova et al., 2002). For all that, little is known on the precise molecular mechanism for the tunicamycin biosynthesis which must account for the biosynthesis of the unusual 11-carbon sugar, tunicamine, and the $\alpha, \beta-1 ", 11^{\prime}$-glycosidic linkage (Tsvetanova et al., 2002). These labeling experiments showed that tunicamine is formed from 6- and 5-carbon carbohydrate precursors, rather than by sequential addition of 2-carbon units.

Here we describe the cloning of the tunicamycins biosynthetic gene cluster by utilization of high-throughput heterologous expression (HHE) strategy combined with bioassay. The production of tunicamycins in heterologous non-producing Streptomyces strains demonstrated the role of 12 genes essential for tunicamycin biosynthesis, thereof leading to a proposed pathway for tunicamycin biosynthesis. Two potential gene clusters related to the biosynthesis of the (potential) tunicamycin-like antibiotics were also identified from Streptomyces clavligerus ATCC 27074 (S. clavligerus hereafter) and Actinosynnema mirum DSM 43827 (A. mirum hereafter). The availability of the tunicamycin biosynthetic gene cluster will provide a foundation for mechanistic investigations into tunicamycin biosynthesis, and pave the way for rational generation of novel tunicamycin derivatives via the strategies of combinatorial biosynthesis and pathway engineering.

\section{RESULTS}

\section{Identification of the tunicamycin biosynthetic gene cluster from $S$. chartreusis}

The unique structural features within tunicamycin render direct cloning of its gene cluster both challenging and unfeasible. Unlike classical natural products including polyketides and nonribosomal peptides, there were no distinctive conserved genes that could be used as optimal probes to screen the genomic library of $S$. chartreusis.

The genome information of S. clavuligerus ATCC27064, a previously reported tunicamycin-like antibiotic (MM 19290) producer (Kenig and Reading, 1979), was released online in 2009. It seems to present us with the potential hopes for the identification of the tunicamycin gene cluster; however, efforts with bioinformatic and genetic analysis of the genome data were unsuccessful because of the paucity of the knowledge on tunicamycin biosynthesis (Price and Tsvetanova, 2007; Medema et al., 2010).

We therefore developed an alternative approach. A HHE strategy combined with bioassay was utilized to screen pJTU2463-derived genomic library (see Experimental Procedures). Three of the S. lividans TK24 recombinants (11C3, 11D8 and 12G1) were identified as showing obvious inhibition against the indicator strain Bacillus subtilis (Fig. S2), indicating the conferred capability of producing bioactive metabolites. Furthermore, the three positive cosmids digested by BamHI were revealed to show significant overlap (Fig. S2). Bioinformatic analysis of the sequenced double ends of the BamHI-derived fragments (cloned in counterpart site of plJ2925) of 11C3 showed that the polypeptides encoded by related open reading frames exhibit high homologies to those in the genome of $S$. clavuligerus (data not shown). These data implied that the three positive cosmids probably possess the entire tunicamycin biosynthetic gene cluster which conferred the TK24 strains with the 
A

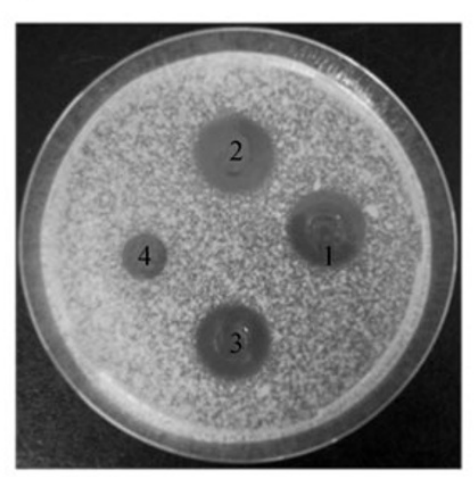

B

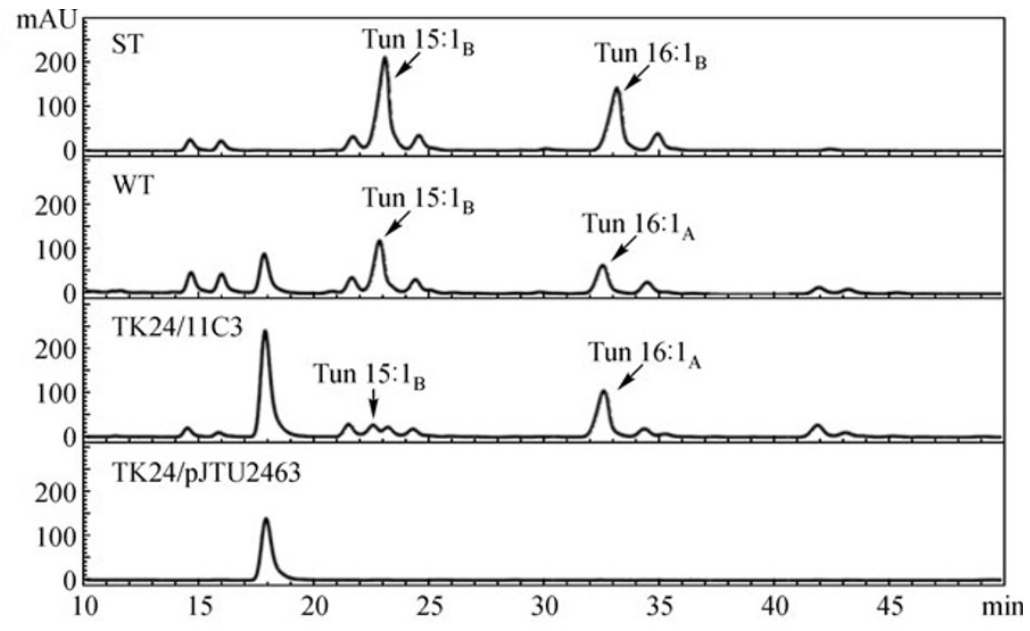

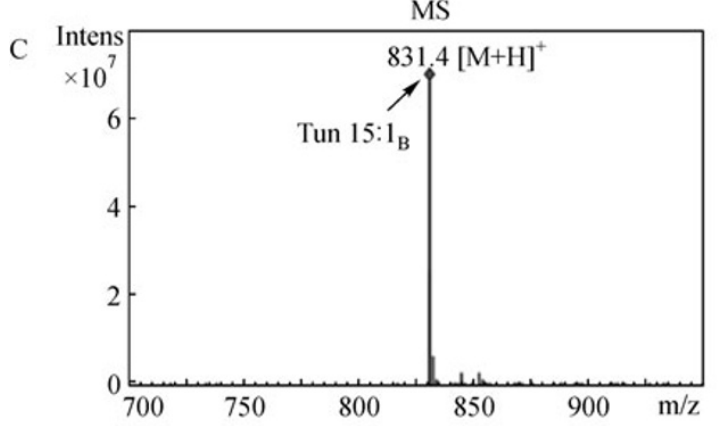

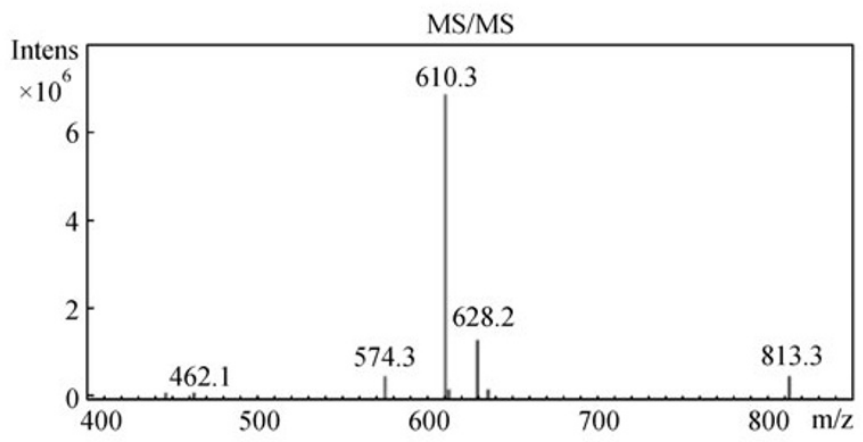

D
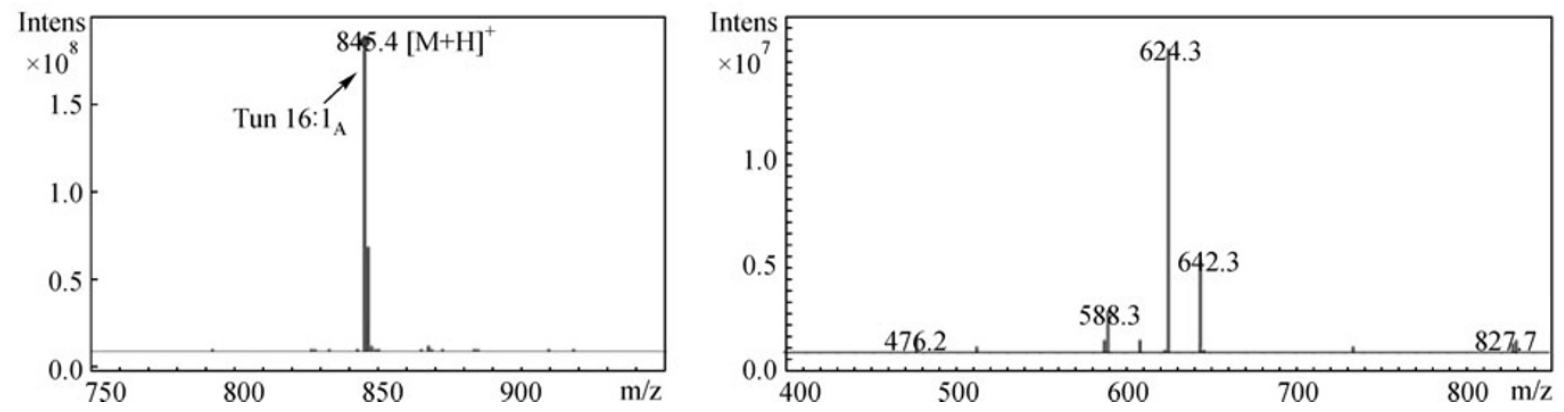

Figure 2. Heterologous production of tunicamycin in S. lividans TK24. (A) Bioassay of the metabolites produced by the TK24 recombinant. Bacillus subtilis was used as indicator strain, and a volume of $40 \mu \mathrm{L}$ samples (except that $20 \mu \mathrm{L} 1.0 \mathrm{mg} / \mathrm{mL}$ of tunicamyicn authentic standard was used in this assay) solved in methanol were used for each assay in this study. Recombinants ( $S$. lividans TK24 carrying 11C3) spotted in (3) was compared with wild-type $S$. chartreusis(2) and tunicamycin authentic standard (1) as a positive controls (1) and S. lividans TK24/pJTU2463 as a negative control (4). (B) HPLC analysis of the metabolites produced by the engineered TK24 recombinant. Tunicamycin authentic standard (ST), and samples from wild-type of $S$. chartreusis (WT), S. lividans TK24/11C3 (TK24/11C3), and S. lividans TK24/pJTU2463 (TK24/pJTU2463), were compared. (C) MS and MS/MS analysis of the Tun 15:1 $1_{B}$ component from the sample of TK24/11C3. The $[\mathrm{M}+\mathrm{H}]^{+}$ion at $m / z$ 831.4 of Tun $15: 1_{B}$ component was fragmented into $610.3,628.2,574.3$, etc. (D) MS and MS/MS analysis of the Tun 16:1 $1_{A}$ component from the sample of TK24/11C3. The [M + H $]^{+}$ ion at $\mathrm{m} / \mathrm{z} 845.4$ of Tun $16: 1_{\mathrm{A}}$ component was fragmented into $624.3,642.3,588.3$, etc. The MS and MS/MS profiles and fragmentation pattern of Tun $15: 1_{\mathrm{B}}$ and Tun $16: 1_{\mathrm{A}}$ of the tunicamycin authentic standard were indicated in Fig. S4. 
capabilities of producing tunicamycin.

\section{Heterologous production of tunicamycin in non-producer S. lividans TK24}

To demonstrate that the TK24 recombinants (individually containing 11C3, 11D8 and 12G1) could make tunicamycins, they were inoculated in TSBY liquid medium for fermentation. Samples of the TK24 recombinants were prepared and subjected for the bioassay. These results indicated that all three of the TK24 recombinants display obvious bioactivity against the indicator strain Bacillus subtilis, in full agreement with the previous bioassay experiments. Moreover, the sample of the TK24/11C3 showed similar bioactivity against the indicator stain to that of wild type S. chartreusis (Fig. 2A).

For further identification of the bioactive metabolites produced by the TK24 recombinants (independently containing 11C3, 11D8 and 12G1) as tunicamycin, extracts of the TK24 recombinants were subjected for HPLC analysis. The results confirmed that the TK24 recombinants gave rise to characteristic tunicamycin peaks with retention times of 22.6 min (Tun 15:1 $1_{\mathrm{B}}$ ) and 32.6 min (Tun 16:1 ${ }_{\mathrm{A}}$ ) (Fig. 2B and Fig. S3). These corresponded to HPLC peaks produced by the tunicamycin authentic standard, and the sample from wild type $S$. chartreusis, while the negative control, S. lividans TK24/pJTU2463 lacked these characteristic peaks (Fig. 2B). Furthermore, the ratio of the major components of Tun 15:1 $\mathrm{B}$ and Tun $16: 1_{\mathrm{A}}$ is ca. 3:2, whereas the major component produced by the TK24/11C3 recombinant was shown to be Tun $16: 1_{\mathrm{A}}$, whose production is $17.0 \mu \mathrm{g} / \mathrm{mL}$ (crude broth). Hence this is 1.56 fold more than that produced by the wild type strain.

To confirm the identities of the tunicamycin components, the two peaks of the TK24/11C3 sample and tunicamycin authentic standard were independently subjected for MS and MS/MS assessment. The results indicated that the peak of Tun $15: 1_{\mathrm{B}}$ give a $[\mathrm{M}+\mathrm{H}]^{+}$ion at $\mathrm{m} / \mathrm{z}$ 831.4, which was fragmented into the ions at $\mathrm{m} / \mathrm{z} 610.3,628.2,574.3$, and 462.1 (Fig. 2C). Likewise, the Tun $16: 1_{\mathrm{A}}$ peak generated a [M $+\mathrm{H}^{+}$ion at $\mathrm{m} / \mathrm{z} 845.4$, and MS/MS analysis showed that the main fragment ions were at $624.3,642.3,588.3$, and 476.2 (Fig. 2D). The 14-Da mass difference between these two sets of ions is due to the $-\mathrm{CH}_{2}$ - difference between $\mathrm{C}_{16: 1}$ and $\mathrm{C}_{15: 1}$ $\mathrm{N}$-acyl chains. Moreover, all of these ions were fully consistent with the MS and MS/MS pattern of the two peaks of the tunicamycin authentic standard (Fig. S4), unambiguously demonstrating the identities of the two components produced by TK24/11C3 recombinants as Tun $15: 1_{\mathrm{B}}$ and Tun $16: 1_{\text {A. }}$.

\section{LC/MS and Q-TOF MS analysis of the tunicamycin profiles of related Streptomcyes recombinants}

To see if $11 \mathrm{C} 3$ could confer other Streptomyces (S. avermitilis and $S$. albus) with the capability of producing variable tunicamycin profiles, extracts of the two recombinants were subjected for bioassay and HPLC analysis. These results showed that the extracts of the recombinants were able to display similar bioactivities against the indicator strain, Bacillus subtilis, to that of TK24 recombinant. Moreover, HPLC results also showed somewhat variable tunicamycin profiles among the three extracts (Fig. S5).

MS/MS fragmentation of tunicamycins has been detailed previously (Tsvetanova and Price, 2001; Tsvetanova et al., 2002). Fragmentation of molecular adduct ions give rise to [M$\left.2^{221}\right]^{+}$ions by neutral loss of the $\mathrm{N}$-acetylglucosaminyl group (Fig. S4). Because these retain the $\mathrm{N}$-acyl substituent, these are characteristic of individual tunicamycins. Further fragmentation involves a neutral loss of the uracil motif and crossring fragmentation of the tunicamine sugar backbone, to generate an $\mathrm{N}$-acyloxy ion that is characteristic of the tunicamycin $\mathrm{N}$-acyl group (Tsvetanova et al., 2002). Hence, $\mathrm{m} / \mathrm{z} 638,624,610$, and 596 are characteristic of Tun17:1, Tun16:1, Tun15:1, and Tun14:1, respectively, and give rise to corresponding $N$-acyloxy ions at $m / z 251,237,223$, and 209. These masses correspond to unsaturated $C_{17: 1}-C_{14: 1}$ acyl chains, as shown in Fig. 1. Selective ion monitoring of these ions established the tunicamycin profiles for the transconjugate strains (Fig. S6).

\section{Sequence analysis of the tunicamycin gene cluster}

A contiguous 37,699-bp region arising from sequencing the positive cosmid 11C3 (GenBank accession number HQ111437), harbors G + C content of $67.81 \%$ (while a Sacl fragment containing the minimal tunicamycin gene cluster contains G + C content of $64.92 \%$ ) (Fig. S7), somewhat lower than that of the typical Streptomyces genomes exemplified by Streptomyces coelicolor A3(2) (Bentley et al., 2002) and Streptomycs avermitilis (Omura et al., 2001), implying the probable non-actinomycial origin of the tunicamycin gene cluster. Frameplot 3.0 Beta online program analysis revealed 39 complete Open Reading Frames (orfs) in sequenced region (cosmid 11C3) as shown in Table 1, Fig. 3 and Fig S7. Of them, 12 orfs (designated tunA-tunL) in one transcriptional unit constitute the gene cluster involved in tunicamycin biosynthesis (Fig. 3).

As proposed, the tunicamycin biosynthetic gene cluster would start with $\operatorname{tun} A$, which encodes a probable NADdependent epimerase/dehydratase. This gene shows high homology ( $42 \%$ identity, $58 \%$ positives) to SSAG-01084 of Streptomyces sp. Mg1. The second gene, tunB, encodes a protein exhibiting homology (32\% identity, $48 \%$ positives) to Htur_3864, a radical SAM domain protein of Haloterrigena turkmenica DSM 5511. Furthermore, TunB also shows homology with $33 \%$ identity to coenzyme $P Q Q$ synthesis protein E (PqqE) of Mycobacterium abscessus ATCC 19977. This family of enzymes typically uses a radical-catalyzed 
Table 1 Deduced functions of the open reading frames in the tun gene cluster

\begin{tabular}{|c|c|c|c|c|c|}
\hline$\overline{\text { protein }}$ & aa & proposed function & homolog, origin & $\begin{array}{c}\text { identity, } \\
\text { similarity (\%) }\end{array}$ & $\begin{array}{l}\text { accession } \\
\text { no. }\end{array}$ \\
\hline $\bar{A}$ & 321 & $\begin{array}{c}\text { NAD-dependent } \\
\text { epimerase/dehydratase }\end{array}$ & $\begin{array}{c}\text { SSAG_01084, Streptomyces sp. } \\
\text { Mg1 }\end{array}$ & 42,58 & ZP_04996782.1 \\
\hline B & 338 & radical SAM protein & $\begin{array}{l}\text { Htur_3864, Haloterrigena } \\
\text { turkmenica DSM } 5511\end{array}$ & 32,48 & YP_003405396.1 \\
\hline C & 318 & $\begin{array}{c}\text { GCN5-related } \\
\text { N-acetyltransferase }\end{array}$ & $\begin{array}{c}\text { Fnod_1042, Fervidobacterium } \\
\text { nodosum Rt17-B1 }\end{array}$ & 33,50 & YP_001410548.1 \\
\hline $\mathrm{D}$ & 472 & glycosyl transferase & $\begin{array}{c}\text { Mmc1_1923, Magnetococcus } \\
\text { sp. MC-1 }\end{array}$ & 26,42 & YP_865837.1 \\
\hline E & 234 & de-N-acetylase & $\begin{array}{c}\text { CRC_02908, } \\
\text { Cylindrospermopsis raciborskii } \\
\text { CS-505 }\end{array}$ & 43,57 & ZP_06309433.1 \\
\hline $\mathrm{F}$ & 327 & UDPglucose 4-epimerase & $\begin{array}{l}\text { POTG_01647, Paenibacillus sp. } \\
\text { oral taxon } 786 \text { str. D14 }\end{array}$ & 46,63 & ZP_04852226.1 \\
\hline G & 203 & phosphoglycerate mutase & Francci3_2350, Frankia sp. Ccl3 & 29,47 & YP_481446.1 \\
\hline $\mathrm{H}$ & 515 & pyrophosphatase & $\begin{array}{l}\text { Bcep18194_A6493, } \\
\text { Burkholderia sp. } 383\end{array}$ & 35,50 & YP_370731.1 \\
\hline I & 304 & $A B C$ transporter & $\begin{array}{c}\text { SBI_04506, S. bingchenggensis } \\
\text { BCW-1 }\end{array}$ & 61,75 & ADI07626.1 \\
\hline $\mathrm{J}$ & 262 & ABC-2 transporter & $\begin{array}{c}\text { Tter_0475, Thermobaculum } \\
\text { terrenum ATCC BAA-798 }\end{array}$ & 32,51 & YP_003322218.1 \\
\hline K & 81 & acyl carrier protein & $\begin{array}{c}\text { Tbis_2371, Thermobispora } \\
\text { bispora DSM } 43833\end{array}$ & 39,58 & YP_003652969.1 \\
\hline L & 229 & $\begin{array}{l}\text { PA-phosphatase related } \\
\text { protein }\end{array}$ & $\begin{array}{c}\text { MicauDRAFT_2890, } \\
\text { Micromonospora aurantiaca } \\
\text { ATCC } 27029\end{array}$ & 33,42 & ZP_06217896.1 \\
\hline
\end{tabular}

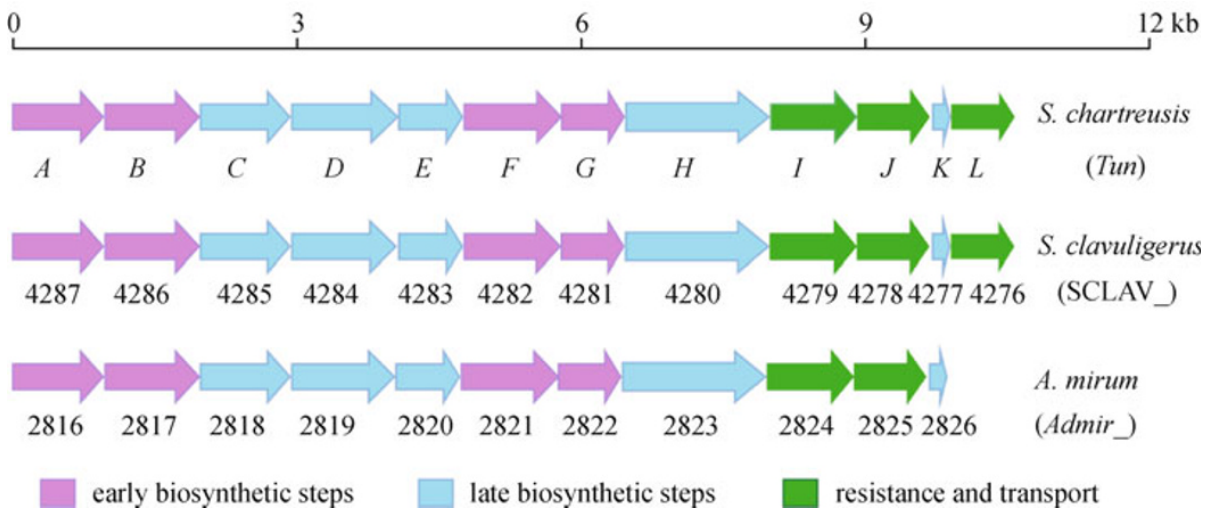

Figure 3. Genetic organization of the complete/partial tunicamycin biosynthetic gene clusters. The proposed functions of the tun genes were described in Table 1. The gene loci located in genomes of $S$. clavuligerus and $A$. mirum were utilized to nominate the target genes for (potential) tunicamycin biosynthesis.

mechanism to generate de novo carbon-carbon bonds. The third gene in the cluster, tunC encodes an enzyme showing 33\% identity to Fond_1042, a GCN5-related $\mathrm{N}$-acetyltransfrase of Fervidobacterium nodosum Rt17-B1 with broad substrates specificity. TunD shows weak homology (26\% identity) to Mmc1_1923 of Magnetococcus sp. MC-
1 , which probably functions as a glyosyltransferase. Based on homology, it seems to be a unique class of glycosyltransferase with as yet uncharacterized catalytical mechanism. The gene tunE encodes a protein homologous to $\mathrm{N}$-acetylglucosaminyl-phosphatidylinositol de- $\mathrm{N}$-acetylase ( $\mathrm{LmbE}$ ) family protein of Cylindrospermopsis raciborskii CS-505. TunE 


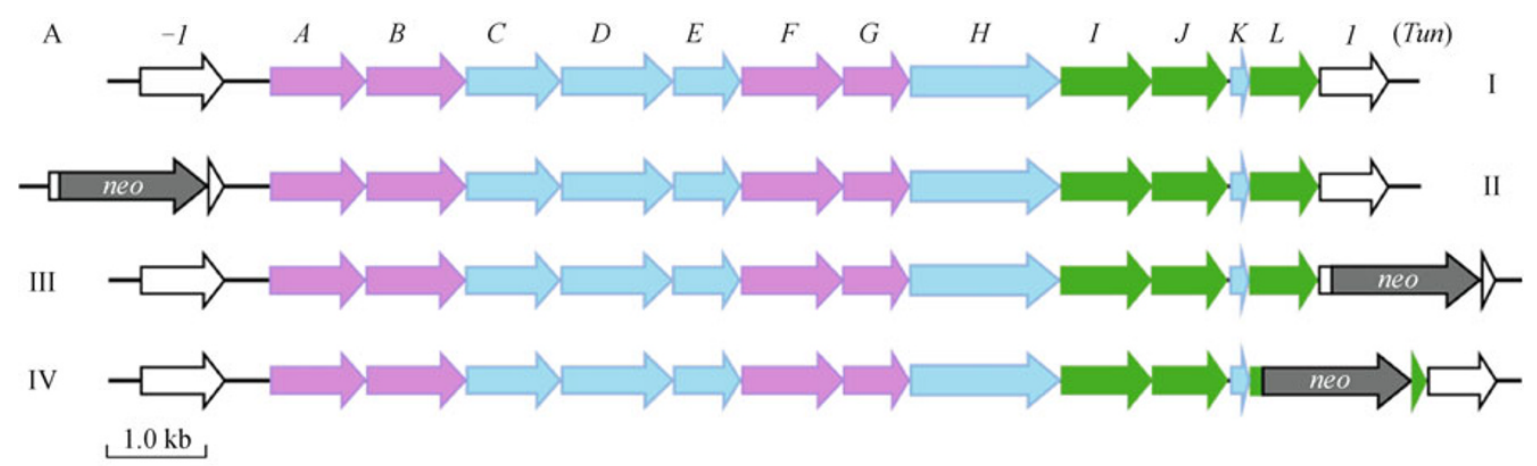

B

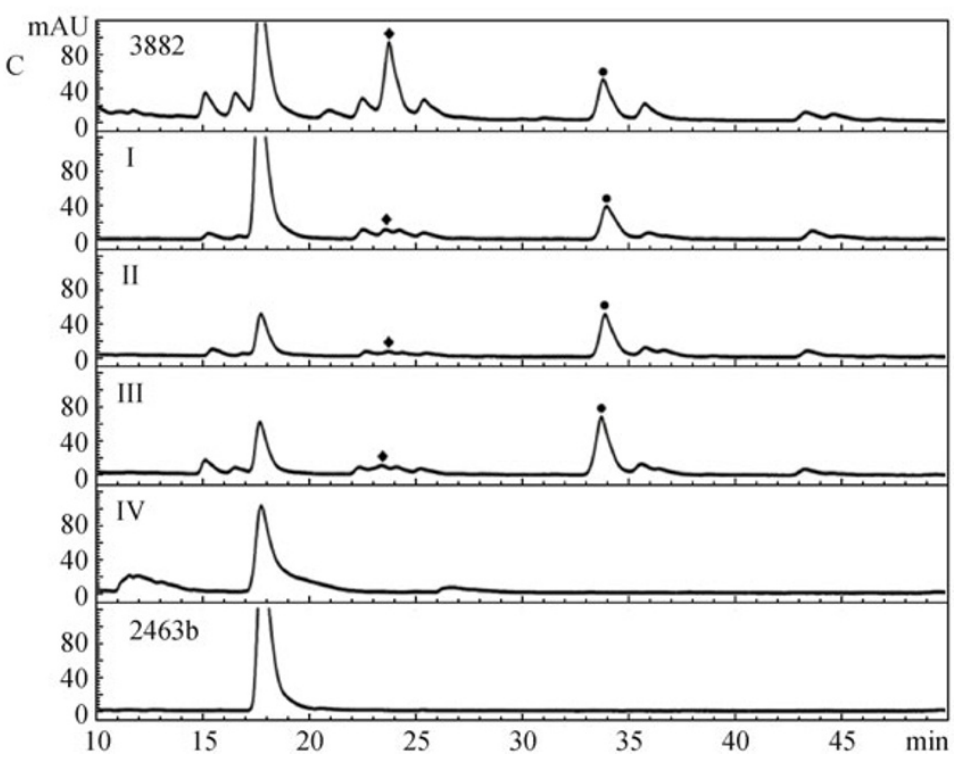

Figure 4. Determination of the minimal gene cluster of tunicamycin. (A) Schematic representation for the mutation of related gene so as to determine the boundaries of tunicamycin gene cluster. I: pJTU5751 wild type, II: pJTU5751 derivative with orf-1 disrupted by neo, III: pJTU5751 derivative with orf1 disrupted by neo, IV: pJTU5751 derivative with tunL disrupted by neo. (B) Bioassay of the metabolites produced by relevant TK24 recombinants carrying pJTU5751 with corresponding mutation in target genes. TK24 recombinant containing intact pJTU5751 was used as positive control (spot 1), and 2463b as negative control (spot 5). TK24 recombinants individually containing pJTU5751 with counterpart mutation of orf-1, tunL and orf1 were indicated as spots 2, 4, and 3, respectively. (C) HPLC analysis of the metabolites produced by related TK24 recombinants. Wild-type of S. chartreusis (3882) and TK24 recombinant carrying pJTU5751 (I) were used as positive controls, and samples (II), (III) and (IV) correspond to those indicated by Fig. 4A. Sample of TK24 recombinant bearing pJTU2463b (2463b) was used as negative control. ( $\bullet)$ : Tun $15: 1_{\mathrm{B}},(\bullet)$ : Tun $16: 1_{\mathrm{A}}$.

protein is therefore deduced to be responsible for removal of the tunicamine-uracil $\mathrm{N}$-acetyl group, prior to addition of the $\mathrm{N}$-acylsubstituent. TunF displays significant homology $(46 \%$ identity, 63\% positives) with POTG_01647, a UDPglucose 4epimerase, of Frankia. The next gene, tunG, encodes a 203aa protein that harbors $29 \%$ identity with the Francci3_2350 of Frankia sp. Ccl3, which is likely to be a CobC-type alpharibazole-5'-phosphate phosphatase. The putative TunH enzyme also exhibits certain homology (35\% identity) to Bcep18194_A6439 of Burkholderia sp. 383, which has been proposed to be a Type I phosphodiesterase/ nucleotidepyrophosphatase. As a result, TunH probably functions as a pyrophosphosphatase in the tunicamycin biosynthetic pathway.

Four genes tunl-tunL are sequentially located downstream of tunH (Fig. 3). Of these, tunl encodes a 304-aa protein with $61 \%$ identity to an ABC transporter (SBI_04506) of Streptomyces bingchenggensis. The protein encoded by tunJ presents $32 \%$ identity of homology to Tter_0475, an ABC-2 transporter of Thermobaculum terrenum ATCC BAA-798. More interestingly, the two genes (tunl and tunJ) correspondingly show homologies matched to the $\mathrm{N}$ terminus and $\mathrm{C}$ terminus of the protein encoded by mtrA (mithramycin resistance gene). As a result, we propose that these two 
genes are involved in the self-resistance and transport of tunicamycin. According to bioinformatic analysis, tunK encodes an 81-aa distinctive protein, which exhibits homology (39\% identity, $58 \%$ positives) to an acyl carrier protein (ACP) of Thermobispora bispora DSM 43833, implicating its unique role in the biosynthesis of tunicamycins. The gene tunL situated in the terminal of the tunicamycin gene cluster encodes a protein resembling a phosphoesterase PAphosphatase-related protein (MicauDRAFT_2890) of Micromonospora aurantiaca ATCC 27029 with $33 \%$ identity. This protein is a super-family of phosphatases and haloperoxidases, which may act as a membrane-associated lipid phosphatase.

\section{Determination of the minimal gene cluster essential for tunicamycin biosynthesis}

According to bioinformatic analysis, tunA-tunL constitutes one operon, suggesting that orf- 1 is probably the left boundary of the tunicamycin gene cluster. For further confirmation, orf-1 was directly disrupted in pJTU5751 by utilization of PCR-targeting technology (Gust et al., 2003), and the resultant pJTU5751 derivative (orf-1 mutated) (Fig. 4A) was introduced into S. lividans TK24 for heterologous expression. After fermentation, extract of the recombinant was subjected for bioassay and LC/MS analysis, and the results showed that the extract of the TK24 recombinant was capable of both showing obvious bioactivity against Bacillus subtilis, and generating the distinctive peaks corresponding to those of the authentic standards of Tun $15: 1_{B}$ and Tun $16: 1_{\mathrm{A}}$ (Fig. 4B and $4 \mathrm{C}$ ), undoubtedly demonstrating that orf-1 is not essential for tunicamycin biosynthesis. Moreover, based on the methods described as above, tunL and orf1 were individually disrupted (Fig. 4A), and the generated phenotypes of resultant TK24 recombinants (bearing pJTU5751 derivatives with orf1 or tunL disrupted) were investigated. The results indicated that mutation of orf1 results in no influence on tunicamycin production (Fig. 4B and $4 \mathrm{C}$ ), however, target inactivation of tunL directly leads to abolishment of tunicamycin production as indicated by bioassay and HPLC analysis (Fig. 4B and 4C). These data unambiguously define that tunA-tunL constitutes the minimal gene cluster of tunicamycin.

\section{Comparative analysis of the (potential) gene clusters for tunicamycin-like family antibiotics biosynthesis in actinomycetes}

From Blast searches and genome mining, two potential tunicamycin-like gene clusters were individually identified in the published genomes of S. clavuligerus 27064 and $A$. mirum DSM 43827 (Fig. 2). S. clavuligerus was previously reported to be capable of making a tunicamycin-like antibiotic (MM 19290) (Kenig and Reading, 1979) which harbors the same backbone as tunicamycin except for the attachment of different $N$-linked acyl chains, while the strain $A$. mirum DSM 43827 was not previously identified as a producer of tunicamycin-like antibiotics. Initially, several $A$. mirum strains including the DSM43827 were fermented to isolate the potential tunicamycin-like antibiotics; however, these efforts were subsequently demonstrated to be negative (data not shown).

Bioinformatic analysis revealed that the three gene clusters have identical genetic organization as indicated in Fig. 3 . Interestingly, the tunL homolog was missing in the potential gene cluster of $A$. mirum DSM 43827, which may partly account for the lack of tunicamycin production by this strain. In addition, the sequence homologies among the three gene clusters were relatively low (Table S3) compared with the gene clusters of validamycin, which was produced by several Streptomyces, for example (Yu et al., 2005; Bai et al., 2006; Jian et al., 2006; Singh et al., 2006). Remarkably, there are no discernable nucleotide homologies in two tunicamycin gene clusters of $S$. chartreusis and $S$. clavuligerus (Table S3).

\section{In-frame deletion of tunA directly results in abolishment of tunicamycin production}

The gene tunA represents the start of the tunicamycin gene cluster. To obtain genetic evidence that tunA was indispensible for tunicamycin biosynthesis, this gene in pJTU5751 was mutated by in-frame deletion with PCR targeting technology (Gust et al., 2003) (Fig. 5A). After that, the resultant pJTU5751/AtunA was conjugated into S. lividans TK24, and the transconjugants validated by PCR were inoculated for further fermentation tests.

As the bioassay results showed, the extracts of the TK24 recombinants containing the pJTU5751/AtunA and the negative control (2463b) have lost bioactivity against Bacillus subtilis, whereas the positive control (TK24/pJTU5751) displays distinct inhibition against the indicator strain (Fig. 5C). Additionally, HPLC analysis results showed that the recombinant TK24/ pJTU5751/AtunA did not yield the distinctive peaks of Tun $15: 1_{B}$ and Tun 16:1 $1_{A}$ when compared with positive controls (Fig. 5D). Hence, these data clearly establish that tunA is essential for tunicamycin biosynthesis.

\section{DISCUSSION}

The use of tunicamycin to inhibit protein glycosylation or bacterial peptidoglycan biosynthesis is well documented in over 5000 citations. The chemical structure and potent bioactivities are known (Tsvetanova et al., 2002; Price and Tsvetanova, 2007), but the precise molecular mechanism for tunicamycin biosynthesis has remained obscure for decades. Indeed, cloning and characterization of the tunicamycin gene cluster not only provides insights into the tunicamycin 

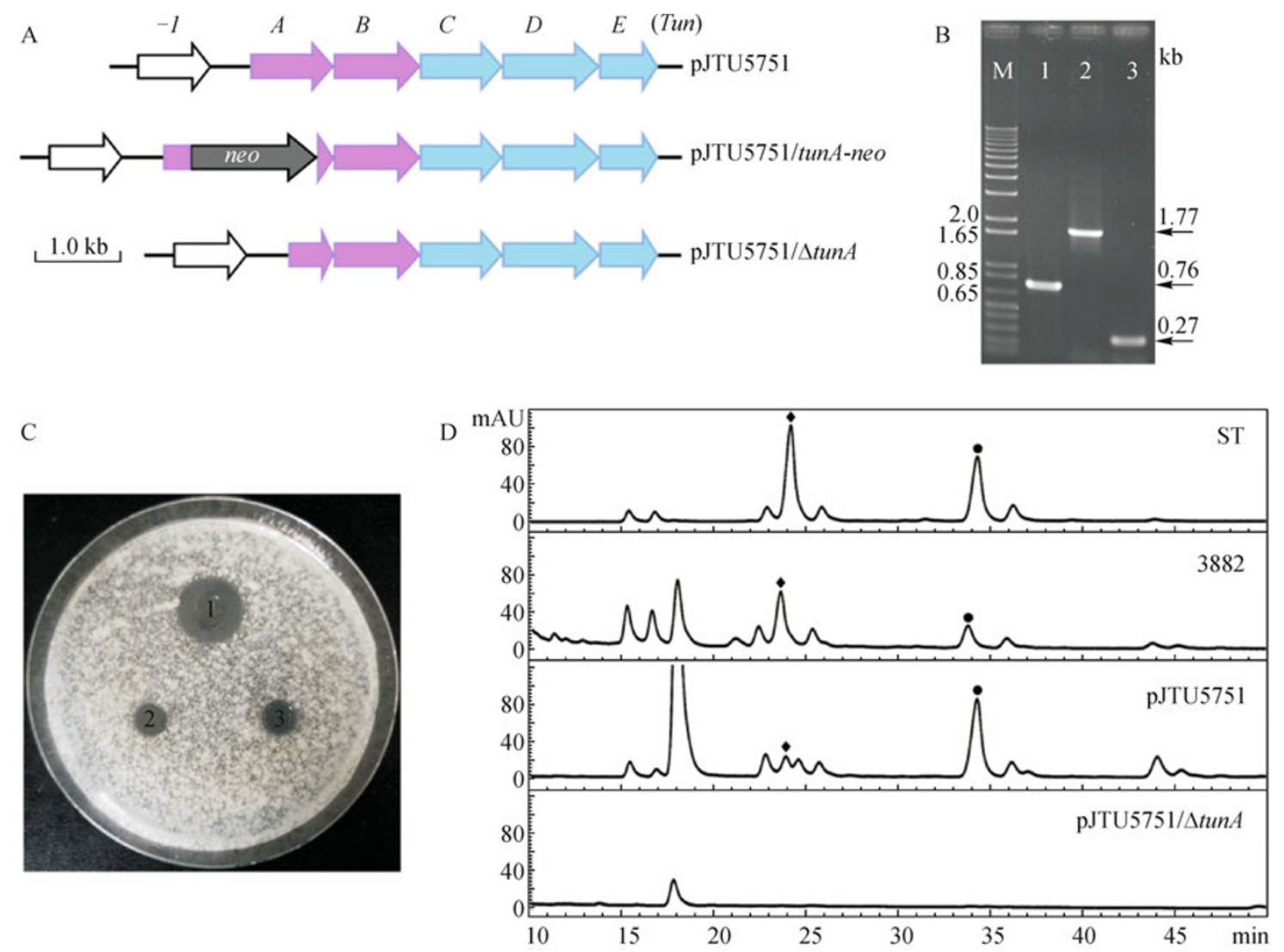

Figure 5. In-frame deletion of tunA. (A) Schematic representation for the in-frame deletion of tunA in pJTU5751. pJTU5751/tunAneo means that the neo cassette replaces 489-bp region of tunA in pJTU5751, pJTU5751/AtunA indicated that the neo cassette was removed by Xbal-Spel double digestion with leaving of an $n$-frame deletion scar. (B) PCR validation of the tunA mutation in pJTU5751, as the neo cassette was recombined into tunA, pJTU5751/tunA-neo would give a ca. 1.77-kb PCR product, while the pJTU5751 shows $0.76-k b$ PCR product; and the PCR product of pJTU5751/AtunA is $0.27-\mathrm{kb}$ in size. (C) Bioassay of the metabolites produced TK24 recombinants carrying pJTU5751/AtunA. (D) The authentic tunicamycin standard (ST), samples of S. chartreusiswild-type (3882) as well as TK24 recombinants independently containing pJTU5751 and pJTU5751/DtunA were compared. $(\bullet)$ : Tun $15: 1_{\mathrm{B}},(\bullet)$ : Tun 16:1 $\mathrm{A}$.

biosynthetic pathway, but may also provide the basis for combinatorial biosynthesis of novel tunicamycin analogs with improved/selective bioactivity.

Earlier metabolic feeding experiments have established that tunicamycin biosynthesis is initiated by utilization of uridine and UDP-GIcNAc as starting substrates. The latter is sequentially converted into the sugar nucleotide UDP. GalNAc-5,6-ene, while uridine was deduced to be oxidized to form uridine-5'-aldehyde as the acceptor for ligation to the UDP-GalNAc-5,6-ene (Tsvetanova et al., 2002; Price and Tsvetanova, 2007).

In the present paper, this mechanism is shown to be essentially correct. Four enzymes, including TunA (dehydratase), TunB (radical SAM protein), TunF (epimerase) and TunG (mutase) are shown to be involved in early biosynthetic steps of tunicamycin. TunA was deduced to utilize UDPGIcNAc to form UDP-4-keto-GIcNAc-5,6-ene, and then this intermediate is further modified to UDP-GIcNAc-5,6-ene under control of TunF (Fig. 6A). Similar studies have previously reported that a biofunctional UDP-GIcNAc C-6 dehydratase/C4 epimerase, FlaA1, from Helicobacter pylori catalyzes sequential conversion of UDP-GIcNAc to UDP-4keto-6-methyl-GlcNAc, which is stereospecifically reduced to UDP-QuiNAc (Creuzenet et al., 2000). Similarly, a wellcharacterized dTDP-glucose 4,6-dehydratase (RffG) from $E$. coli uses dTDP-glucose to generate dTDP-4-keto-6-deoxyglucose. More importantly, this enzyme generates an intermediate, dTDP-4-keto-glucose-5,6-ene (Gross et al., 2000), which is analogous to the corresponding intermediate currently proposed in the tunicamycin biosynthetic pathway.

It is tempting to propose that the radical SAM protein homolog TunB enzyme catalyzes the formation of 5 '-uridine radical from uridine, which then reacts with UDP-4-keto-6deoxy-GIcNAc to form a carbon-carbon bond at the C-6' 

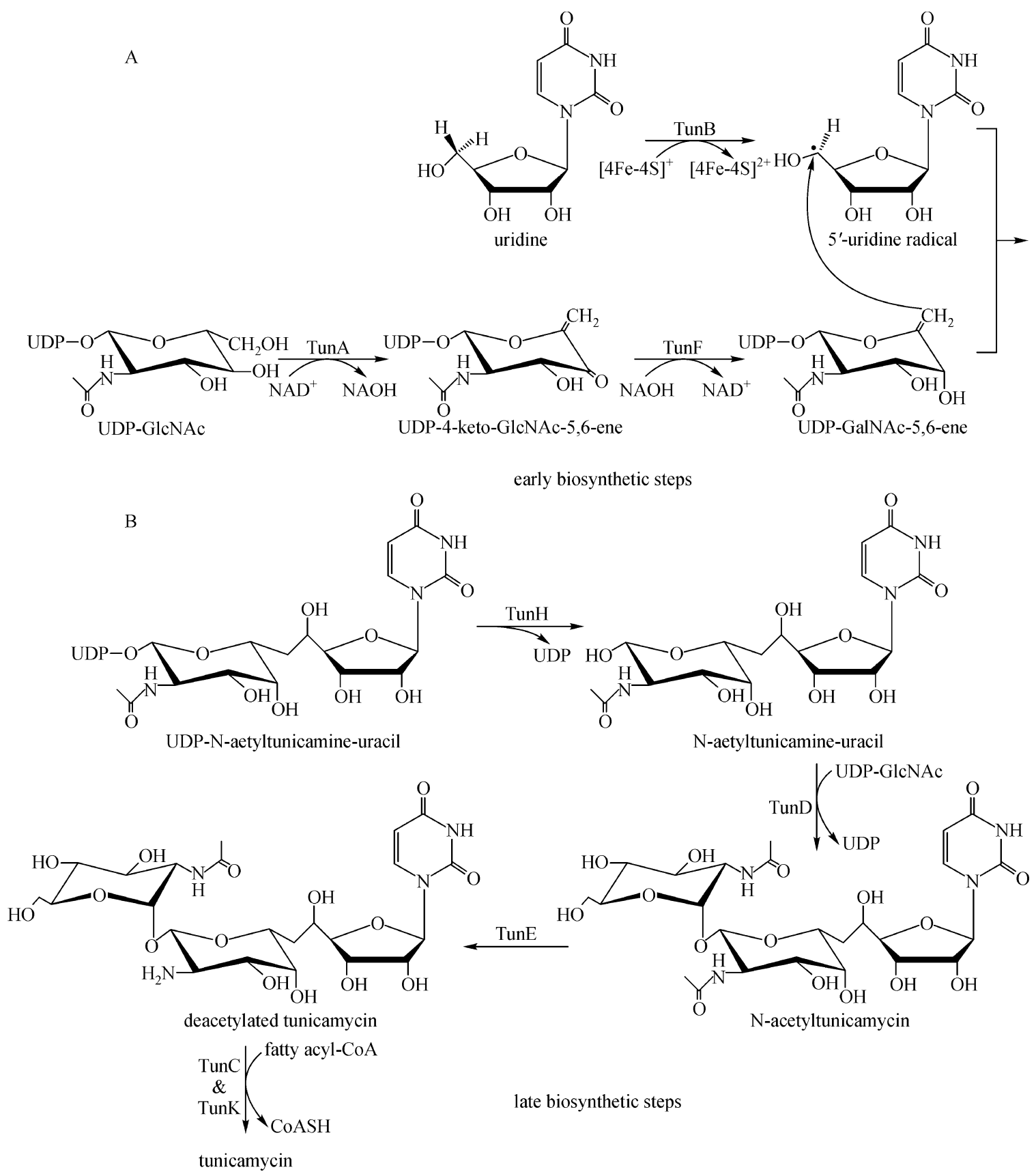

Figure 6. Proposed pathway for tunicamycin biosynthesis. (A) Early biosynthetic steps for tunicamycin biosynthesis, and the late steps for tunicamycin biosynthesis are indicted as (B).

position (Fig. 6A). A radical SAM protein (PqqE) homologous to TunB from Klebsiella pneumoniae is capable of catalyzing carbon-carbon bond formation in the biosynthesis of coenzyme PQQ (Wecksler et al., 2009). However, to the best of our knowledge this mechanism has not been previously observed for carbohydrate metabolism. Related to this, TunG, a putative phosphoglycerate mutase with homology to Francci3_2350, is potentially a CobC-type alpha-ribazole-5'phosphate phosphatase. CobC is involved in the biosynthesis of cobalamin (vitamin B12), wherein the dephosphorylation of adenosylcobalamin- 5 '-phosphate by the CobC phosphatase is the last step of the pathway (Zayas and Escalante-Semerena, 2007). Since cobalamin is a co-factor for the radical SAM proteins (Tun B), it is quite possible that the TunG promotes the production of cobalamin for its use during the biosynthesis of tumicamycin.

The later steps for tunicamycin biosynthesis are also proposed based on the feeding experiments with 
isotope-labeled substrates (Tsvetanova et al., 2002; Price and Tsvetanova, 2007). The initial reaction involves the hydrolysis of UDP from UDP- $N$-acetyltuniamine uracil to give $\mathrm{N}$-acetyltunicamine uracil. This then acts as the acceptor substrate for addition of the $\alpha, \beta-1$ ", 1 '-GIcNAc residue, after which tunicamyin is bio-synthesized by stepwise reactions of deacetylation and transacylation (Price and Tsvetanova, 2007). Our analysis of the tunicamycin biosynthetic gene cluster identified five putative proteins: TunC (acetyltransferase), TunD (glycosyltransferase), TunE (deacetylase), TunH (nucleotide pyrophosphatase) and TunK (ACP), as candidate enzymes responsible for the late steps for tunicamycin biosynthesis. These late biosynthetic steps are initiated by TunH catalyzing the conversion of UDP- $N$-acetyltunicamine uracil to $N$-acetyltunicamine uracil. This subsequently acts as the glycosidic acceptor for addition of an $\alpha, \beta-1 ", 1^{\prime}$-linked GIcNAc residue from the donor molecule UDP-GIcNAc, as catalyzed by TunD (Fig. 6B). Structurally, the $\alpha, \beta-1 ", 11^{\prime}-$ glycosidic-glycosidic linkage is highly unusual and, additionally, TunD displays only marginal homology to known glycosyl transferase family protein, suggesting that this enzyme harbors an unprecedented catalytical mechanism. The intermediate $\mathrm{N}$-acetyltunicamycin is subsequently deacetylated by TunE to deacetylated tunicamycin (Fig. 6B). TunC and TunK are proposed as the targeted enzymes essential for acyl chains transfer and $\mathrm{N}$-acylation. We further propose that TunC is responsible for independently selecting different fatty acyl CoA and transferring them to TunK (Fig. 6B). The naturally-occurring tunicamycins can exhibit various $N$-linked acyl chains, suggesting that the $\mathrm{N}$-acyl transferase, TunC, has similarly broad substrate flexibility. Despite gaining insight into the tunicamycin biosynthetic gene cluster, we were unable to identify an obvious candidate gene responsible for the amide bond formation by homology alone, but it may be distributed in different loci of the genome as reported for several previous examples of biosynthesis (Yu et al., 2002; Ostash et al., 2007; Chen et al., 2009). However, the location of the the small protein encoded by tunK in the tunicamycin gene cluster suggests a potential role for this enzyme for the amide bond formation (Fig. 6B). Moreover, the genes governing biosynthesis of the fatty acyl chains were missing from the tunicamycin gene cluster, as reported for the caprazamycin and liposidomycin biosynthetic pathways (Kaysser et al., 2009, 2010). Accordingly, it is reasonable to believe the fatty acyl chains are derived from primary metabolic pathways, and manipulation of the fatty acid biosynthetic pathway would be of great potential to generate novel tunicamycin derivatives.

Investigation of the tunicamycin biosynthetic gene cluster also identified three proteins with a potential role in a mechanism of self-resistance. Tunl (ABC transporter, ATP binding protein), TunJ (ABC-2 transporter) and TunL (phosphatase) were assigned as the functions responsible for the resistance and transport of tunicamycin. Recent research on the crystal structure of the TmrD tunicamycin resistance protein revealed that it is more likely a kinase (Kapp et al., 2008) rather than an ATP-dependent transporter $(\mathrm{TmrB})$ as previously described by Noda et al. (1992, 1995). Accordingly, it is proposed that Tunl functions as ATPase that inactivates tunicamycin via phosphorylation, which is subsequently transported and dephosphorylated by TunJ and TunL.

In summary, we report the finding and functional analysis of the tunicamycin biosynthetic gene cluster. Twelve contiguous genes, tunA-L, have been identified from a known tunicamycin producer, $S$. chartreusis. Nine of these encode for functions implicated in a novel seven step biosynthetic pathway. These include a radical SAM protein (TunB) with a unique role in the formation of the $5^{\prime}-6$ ' carbon-carbon bond in the 11-carbon tunicamine sugar backbone. Another putative protein (TunD) is an unusual glycosyltransferase that forms an anomeric-to-anomeric $\alpha, \beta-1 ", 1^{\prime}$-glycosidic bond. Three other genes (tunJ, $K, L$ ) are implicated in tunicamycin transport and self-resistance. We also identified homologs of this gene cluster in two other organism; S. clavuligerus, a known producer of tunicamycin-like antibiotics, and a nonproducing strain of $A$. mirum. We anticipate that manipulation of the pathway would be of great potential to generate novel tunicamycin-based inhibitors with modified activity and selectivity.

\section{MATERIALS AND METHODS}

\section{Strains, plasmids (cosmids), general methods and culture} conditions

Strains, plasmids (cosmids) used are described in Supplemental Data (Table S1), and PCR primers were listed in the Table S2. Streptomyces and its derivatives were grown at $30^{\circ} \mathrm{C}$ on MS agar (Kieser et al., 2000), or in TSBY liquid medium (3\% Difco tryptic soy broth powder, $10.3 \%$ sucrose, $1 \%$ Difco yeast extract, $\mathrm{pH} 7.2$ ). General methods for $E$. coli or Streptomyces manipulation were based on protocol of Sambrook et al. (1989) or Kieser et al. (2000). The final antibiotic concentrations used for selection of $E$. coli or Streptomyces were as follows: ampicillin $100 \mu \mathrm{g} / \mathrm{mL}$, apramycin $30 \mu \mathrm{g} / \mathrm{mL}$, and kanamycin $50 \mu \mathrm{g} / \mathrm{mL}$.

\section{Genomic library construction for S. chartreusis}

Standard methods were adopted for the construction of the pJTU2463 (see Table S1) derived genomic library for S. chartreusis (Sambrook et al., 1989; Kieser et al., 2000) and E. coli XLUZ (Table $\mathrm{S} 1$, Tao et al., unpublished) was selected as suitable host cells.

\section{High-throughput screening of the positive cosmids from genomic library of $S$. chartreusis}

Cosmids of the genomic library of $S$. chartreusis were transferred into S. lividans TK24 with adoption of the method described previously by Martinez et al. (2004). Bacillus subtilis was used as an indicator strain for bioassay screening of the S. lividans TK24 recombinants bearing 
positive cosmids that contained the complete tunicamycin gene cluster (Tsvetanova and Price, 2001).

\section{Nucleotide sequence accession number}

The nucleotide sequence reported in this paper is available in the GenBank database under accession No. HQ111437.

\section{DNA sequencing and sequence analysis}

DNA sequencing was accomplished at Shanghai Major BioTech Co. Ltd using an Applied Biosystems model 3730 automated DNA sequencer, and sequence analysis was carried out with FramePlot 3.0beta online program (http://watson.nih.go.jp/ jun/cgi-bin/frameplot-3.0b.pl) (Ishikawa and Hotta, 1999). DNA and protein homology searches were carried out with PSI-BlastP online software.

\section{Construction of pJTU5751 for mutational analysis of the target tun genes}

For construction of pJTU571, 11C3 cosmid (containing Xbal and Spel sites which were correspondingly located at both sides of foreign insertion) was initially digested by Xbal, and the resultant cohesive ends of linear fragment were blunted by Klenow Fragment (Fermentas). After that, the blunted linear fragment was self-ligated to result in pJTU5751a, in which the Spel site was subsequently blocked with the method described as above.

\section{Direct mutation of tunL (target gene) in the positive cosmid pJTU5751}

For direct mutation of tunL (target gene) in cosmid pJTU5751, Primers TunLtgtF and TunLtgtR (Table S2) were designed to amplify the neo cassette (kanamycin resistance gene from SuperCos 1 ) so as to disrupt tunL via PCR-targeting technology (Gust et al., 2003), and the PCR primers tunLexf and tunLexr (Table S2) were used to validate the tunL mutation. The same method described as above was performed for direct mutation of other target genes in pJTU5751.

\section{In-frame deletion of tunA in pJTU5751}

For in-frame deletion of tunA, a neo cassette from SuperCos 1 was amplified with primers tunAtgtF and tunAtgtR (Table S2), and subsequently recombined into the tunA gene in PJTU5751 to produce pJTU5751/tunA-neo by PCR-targeting technology (Gust et al., 2003). For in-frame deletion of tunA, the neo cassette was removed from pJTU5751/tunA-neo by Xbal-Spel double digestion, and an in-frame deletion scar was left to give rise to pJTU5751/AtunA. The PCR primers tunAexf and tunAexr were designed to identify the tunA mutant.

\section{Tunicamycin production, purification and assay}

S. chartreusis and related Streptomyces recombinants were cultivated in TSBY liquid medium for tunicamycin production. After fermentation at $30^{\circ} \mathrm{C}$ for $5 \mathrm{~d}$, samples were purified according to the method of Tsvetanova and Price (2001). A bioassay and LC/MS
(Agilent 1100 series LC/MSD Trap system) were used for detection of tunicamycin. For the bioassay, Bacillus subtilis was selected as indicator strain, which was grown on LA medium (1.0\% agar). LC/MS analysis was performed on a ZORBA SB-C18 Column (Agilent, $3.5 \mu \mathrm{m}, 2.1 \times 150 \mathrm{~mm}$ ) with an elution gradient of $35 \%-45 \%$ acetonitrile: $0.1 \%$ aqueous acetic acid (HPLC grade) over $50 \mathrm{~min}$ at $0.2 \mathrm{~mL} / \mathrm{min}$. The elution was monitored at $260 \mathrm{~nm}$ with a DAD detector, and the data were analyzed offline with Agilent data software.

\section{Quantification of specific components of tunicamycin}

For the quantification of tunicamycin produced by related strains, commercial tunicamycin (Sigma-Aldrich, St. Louis, IL) was used to generate a standard curve, and the quantity of specific tunicamycin component was calculated based on this curve.

\section{Conditions for LC/MS and MS/MS analysis}

An Agilent 1100 series LC/MSD Trap system with an electrospray ionization source was used for MS analysis of tunicamycin in positive ion detection mode. The parameters for MS analysis are as follows: drying gas flow: $10 \mathrm{~L} / \mathrm{mL}$, nebulizer pressure: $30 \mathrm{psi}$, and drying gas temperature $325^{\circ} \mathrm{C}$. MS/MS fragmentations were obtained using a quadrupole orthogonal time-of-flight (Q-OTOF) mass spectrometer (Applied Biosystems/MDS Sciex QSTAR Elite, Toronto, Canada) with turbospray ionization. Samples were introduced by online RP-HPLC as described with a split flow rate of $10 \mu \mathrm{L} / \mathrm{min}$. Data was processed using Analyst QS 2.0. MALDI-TOF mass spectra were recorded on a Bruker-Daltonic (Billerica, MA) Omniflex instrument operating in reflecton mode using 2,5-dihydrobenzoic acid matrix. Laser excitation was at $337.1 \mathrm{~nm}$, typically at $75 \%$ of $150 \mu \mathrm{J}$ maximum output, and 60 shots were accumulated.

\section{ACKNOWLEDGEMENTS}

We are sincerely grateful to Dr. Hyung-Jin Kwon, Myongji University, Dept. Biologic Sciences, Yongin, S. Korea, for helpful discussion and many valuable comments. We'd also like to acknowledge the help of my NCAUR-ARS-USDA colleagues David Labeda and Michael Bowman of NCAUR-ARS-USDA, Peoria, I.L., who helped with the culturing and MS of the various Actinosynnema strains. This work was supported by the National Basic Research Program (973 Program) and the National Programs for High Technology Research and Development Program (863 Program) from the Ministry of Science and Technology, the National Science Foundation of China, the Ministry of Education, the Science and Technology Commission of Shanghai Municipality, and Shanghai Leading Academic Discipline Project B203.

\section{ABBREVIATIONS}

aa, amino acid; aac(3)IV, apramycin resistance gene; $\mathrm{ABC}$-transporter, ATP binding cassette transporter; ACP, acyl carrier protein; AT, acyl transferase; GalNAc, N-acetylgalactose; GlcNAc, N-acetylglucosamine; GPT, GIcNAc-1-P transferase; HHE, high-throughput heterologous expression; kDa, kilo-dalton; MraY, phospho-MurNAcpentapeptide translocase; neo, neomycin/kanamycin resistance 
gene; oriT, origin of transfer; SAM, S-adenosyl methionine; TmrB (TmrD), tunicamycin resistance protein

\section{REFERENCES}

Bai, L., Li, L., Xu, H., Minagawa, K., Yu, Y., Zhang, Y., Zhou, X., Floss, H.G., Mahmud, T., and Deng, Z. (2006). Functional analysis of the validamycin biosynthetic gene cluster and engineered production of validoxylamine A. Chem Biol 13, 387-397.

Bentley, S.D., Chater, K.F., Cerdeño-Tárraga, A.M., Challis, G.L., Thomson, N.R., James, K.D., Harris, D.E., Quail, M.A., Kieser, H., Harper, D., et al. (2002). Complete genome sequence of the model actinomycete Streptomyces coelicolor A3(2). Nature 417, 141-147.

Chen, W., Huang, T., He, X., Meng, Q., You, D., Bai, L., Li, J., Wu, M., $\mathrm{Li}, \mathrm{R}$., Xie, Z., et al. (2009). Characterization of the polyoxin biosynthetic gene cluster from Streptomyces cacaoi and engineered production of polyoxin $\mathrm{H}$. J Biol Chem 284, 10627-10638.

Creuzenet, C., Belanger, M., Wakarchuk, W.W., and Lam, J.S. (2000). Expression, purification, and biochemical characterization of WbpP, a new UDP-GIcNAc C4 epimerase from Pseudomonas aeruginosa serotype O6. J Biol Chem 275, 19060-19067.

Eckardt, K. (1983). Tunicamycins, streptovirudins, and corynetoxins, a special subclass of nucleoside antibiotics. J Nat Prod 46, 544-550.

Eckardt, K., Thrum, H., Bradler, G., Tonew, E., and Tonew, M. (1975). Streptovirudins, new antibiotics with antibacterial and antiviral activity. II. Isolation, chemical characterization and biological activity of streptovirudins A1, A2, B1, B2, C1, C2, D1, and D2. J Antibiot (Tokyo) 28, 274-279.

Gross, J.W., Hegeman, A.D., Vestling, M.M., and Frey, P.A. (2000). Characterization of enzymatic processes by rapid mix-quench mass spectrometry: the case of dTDP-glucose 4,6-dehydratase. Biochemistry 39, 13633-13640.

Gust, B., Challis, G.L., Fowler, K., Kieser, T., and Chater, K.F. (2003). PCR-targeted Streptomyces gene replacement identifies a protein domain needed for biosynthesis of the sesquiterpene soil odor geosmin. Proc Natl Acad Sci U S A 100, 1541-1546.

Ishikawa, J., and Hotta, K. (1999). FramePlot: a new implementation of the frame analysis for predicting protein-coding regions in bacterial DNA with a high G + C content. FEMS Microbiol Lett 174, 251-253.

Jian, X., Pang, X., Yu, Y., Zhou, X., and Deng, Z. (2006). Identification of genes necessary for jinggangmycin biosynthesis from Streptomyces hygroscopicus 10-22. Antonie Van Leeuwenhoek 90, 29-39.

Kapp, U., Macedo, S., Hall, D.R., Leiros, I., McSweeney, S.M., and Mitchell, E. (2008). Structure of Deinococcus radiodurans tunicamycin-resistance protein $(\mathrm{TmrD})$, a phosphotransferase. Acta Crystallogr Sect F Struct Biol Cryst Commun 64, 479-486.

Kaysser, L., Lutsch, L., Siebenberg, S., Wemakor, E., Kammerer, B., and Gust, B. (2009). Identification and manipulation of the caprazamycin gene cluster lead to new simplified liponucleoside antibiotics and give insights into the biosynthetic pathway. J Biol Chem 284, 14987-14996.

Kaysser, L., Siebenberg, S., Kammerer, B., and Gust, B. (2010). Analysis of the liposidomycin gene cluster leads to the identification of new caprazamycin derivatives. Chembiochem 11, 191-196.
Keenan, R.W., Hamill, R.L., Occolowitz, J.L., and Elbein, A.D. (1981). Biological activities of isolated tunicamycin and streptovirudin fractions. Biochemistry 20, 2968-2973.

Kenig, M., and Reading, C. (1979). Holomycin and an antibiotic (MM 19290) related to tunicamycin, metabolites of Streptomyces clavuligerus. J Antibiot (Tokyo) 32, 549-554.

Kieser, T., Bibb, M.J., Chater, K.F., Butter, M.J., and Hopwood, D.A. (2000). Practical Streptomyces Genetics, 2nd ed., John Innes Foundation, Norwich, United Kingdom.

Kimura, K., and Bugg, T.D. (2003). Recent advances in antimicrobial nucleoside antibiotics targeting cell wall biosynthesis. Nat Prod Rep 20, 252-273.

Liang, X., Lu, Y., Neubert, T.A., and Resh, M.D. (2002). Mass spectrometric analysis of GAP-43/neuromodulin reveals the presence of a variety of fatty acylated species. J Biol Chem 277 , 33032-33040.

Martinez, A., Kolvek, S.J., Yip, C.L., Hopke, J., Brown, K.A., MacNeil, I.A., and Osburne, M.S. (2004). Genetically modified bacterial strains and novel bacterial artificial chromosome shuttle vectors for constructing environmental libraries and detecting heterologous natural products in multiple expression hosts. Appl Environ Microbiol 70, 2452-2463.

Medema, M.H., Trefzer, A., Kovalchuk, A., van den Berg, M., Müller, U., Heijne, W., Wu, L., Alam, M.T., Ronning, C.M., Nierman, W.C., et al. (2010). The sequence of a 1.8-mb bacterial linear plasmid reveals a rich evolutionary reservoir of secondary metabolic pathways. Genome Biol Evol 2, 212-224.

Noda, Y., Takatsuki, A., Yoda, K., and Yamasaki, M. (1995). TmrB protein, which confers resistance to tunicamycin on Bacillus subtilis, binds tunicamycin. Biosci Biotechnol Biochem 59, 321-322.

Noda, Y., Yoda, K., Takatsuki, A., and Yamasaki, M. (1992). TmrB protein, responsible for tunicamycin resistance of Bacillus subtilis, is a novel ATP-binding membrane protein. J Bacteriol 174, 4302-4307.

Omura, S., Ikeda, H., Ishikawa, J., Hanamoto, A., Takahashi, C., Shinose, M., Takahashi, Y., Horikawa, H., Nakazawa, H., Osonoe, T., et al. (2001). Genome sequence of an industrial microorganism Streptomyces avermitilis: deducing the ability of producing secondary metabolites. Proc Natl Acad Sci U S A 98, 12215-12220.

Ostash, B., Saghatelian, A., and Walker, S. (2007). A streamlined metabolic pathway for the biosynthesis of moenomycin A. Chem Biol 14, 257-267.

Patterson, S.I., and Skene, J.H. (1994). Novel inhibitory action of tunicamycin homologues suggests a role for dynamic protein fatty acylation in growth cone-mediated neurite extension. J Cell Biol 124, 521-536.

Price, N.P., and Momany, F.A. (2005). Modeling bacterial UDPHexNAc: polyprenol-P HexNAc-1-P transferases. Glycobiology 15, 29R-42R.

Price, N.P., and Tsvetanova, B. (2007). Biosynthesis of the tunicamycins: a review. J Antibiot (Tokyo) 60, 485-491.

Sambrook, J., Fritsch, E.F., and Maniatis, T. (1989). Molecular Cloning: a Laboratory Manual, 2nd ed., Cold Spring Harbor Laboratory Press, NY.

Singh, D., Seo, M.J., Kwon, H.J., Rajkarnikar, A., Kim, K.R., Kim, S. O., and Suh, J.W. (2006). Genetic localization and heterologous expression of validamycin biosynthetic gene cluster isolated from 
Streptomyces hygroscopicus var. limoneus KCCM 11405 (IFO 12704). Gene 376, 13-23.

Tsvetanova, B.C., Kiemle, D.J., and Price, N.P. (2002). Biosynthesis of tunicamycin and metabolic origin of the 11-carbon dialdose sugar, tunicamine. J Biol Chem 277, 35289-35296.

Tsvetanova, B.C., and Price, N.P. (2001). Liquid chromatographyelectrospray mass spectrometry of tunicamycin-type antibiotics. Anal Biochem 289, 147-156.

Vogel, P., Petterson, D.S., Berry, P.H., Frahn, J.L., Anderton, N., Cockrum, P.A., Edgar, J.A., Jago, M.V., Lanigan, G.W., Payne, A. L., et al. (1981). Isolation of a group of glycolipid toxins from seedheads of annual ryegrass Lolium rigidum Gaud.) infected by Corynebacterium rathayi. Aust J Exp Biol Med Sci 59, 455-467.

Wecksler, S.R., Stoll, S., Tran, H., Magnusson, O.T., Wu, S.P., King, D., Britt, R.D., and Klinman, J.P. (2009). Pyrroloquinoline quinone biogenesis: demonstration that PqqE from Klebsiella pneumoniae is a radical S-adenosyl-L-methionine enzyme. Biochemistry 48 , 10151-10161.

Winn, M., Goss, R.J., Kimura, K., and Bugg, T.D. (2010). Antimicrobial nucleoside antibiotics targeting cell wall assembly: recent advances in structure-function studies and nucleoside biosynthesis. Nat Prod Rep 27, 279-304.

Xu, L., Appell, M., Kennedy, S., Momany, F.A., and Price, N.P. (2004). Conformational analysis of chirally deuterated tunicamycin as an active site probe of UDP-N-acetylhexosamine:polyprenol-P Nacetylhexosamine-1-P translocases. Biochemistry 43, 13248-13255.

Yu, T.W., Bai, L., Clade, D., Hoffmann, D., Toelzer, S., Trinh, K.Q., Xu, J., Moss, S.J., Leistner, E., and Floss, H.G. (2002). The biosynthetic gene cluster of the maytansinoid antitumor agent ansamitocin from Actinosynnema pretiosum. Proc Natl Acad Sci U S A 99, 7968-7973.

Yu, Y., Bai, L., Minagawa, K., Jian, X., Li, L., Li, J., Chen, S., Cao, E., Mahmud, T., Floss, H.G., et al. (2005). Gene cluster responsible for validamycin biosynthesis in Streptomyces hygroscopicus subsp. jinggangensis 5008. Appl Environ Microbiol 71, 5066-5076.

Zayas, C.L., and Escalante-Semerena, J.C. (2007). Reassessment of the late steps of coenzyme B12 synthesis in Salmonella enterica: evidence that dephosphorylation of adenosylcobalamin-5'-phosphate by the CobC phosphatase is the last step of the pathway. $J$ Bacteriol 189, 2210-2218. 